\title{
COMPETENCIA PARTIDISTA Y RENDIMIENTO POLÍTICO ELECTORAL DE LOS PARTIDOS POLÍTICOS MEXICANOS EN ELECCIONES PRESIDENCIALES ENTRE LOS AÑOS DE 1994 Y 2006
}

Luis Eduardo León Ganatios ${ }^{1}$

\author{
Sumario:
}

1. Notas introductorias. 2. Fragmentación y número efectivo de partidos. 3. Concentración de partidos. 4. Volatilidad electoral. 5. Conclusiones. 6. Bibliografía.

\begin{abstract}
Resumen: La competencia partidista, en elecciones presidenciales a nivel federal en México, se ha intensificado a partir del año 2000, con la victoria de un partido distinto al Partido Revolucionario Institucional, por ende, han variado las preferencias electorales y la oferta electoral. La finalidad de este artículo es medir mediante una serie de indicadores como Número efectivo de partidos, Índice de concentración, índice de fragmentación de Rae y volatilidad las posibles variaciones en la tendencia de voto, el sistema de partidos políticos y el cambio de preferencias de un partido hacia otro. Con el fin de aproximar un posible escenario para las elecciones presidenciales de 2012.
\end{abstract}

Palabras Claves: Competencia partidista, número efectivo de partidos, concentración, volatilidad, elecciones.

\begin{abstract}
Party competition in federal elections in Mexico has been intensified since 2000, hence electoral preferences have varied widely. The main purpose of this article is to measure through a series of indicators such as effective number of parties, concentration of parties index and RAE's index the possible variations and shifts in voting tendencies and preferences from one party to another. The article concludes providing possible future scenarios for the 2012 presidential elections.
\end{abstract}

Keywords: Party competition, effective number of parties, concentration, volatility, elections.

\section{NOTAS INTRODUCTORIAS}

La historia política de México durante el siglo XX está determinada por la influencia del Partido Revolucionario Institucional (PRI) dicho partido "contribuyó de manera decisiva a la modernización del país y estabilizar políticamente a un México incendiado por las revueltas postrevolucionarias... Aunque también fracasó en su misión de mejorar las condiciones socioeconómicas de los grupos o clases en desventaja". ${ }^{2}$

\footnotetext{
${ }^{1}$ Doctor por la Universidad de Salamanca. Profesor de carrera de la Universidad de Guanajuato en la división de Derecho, Política y Gobierno.

${ }^{2}$ Báez, Carlos, México en Alcántara Manuel y Freidenberg Flavia (Coord.) Partidos Politicos de América Latina. Fondo de cultura económica (México), 2003 p. 382.
} 
Un partido fuertemente institucionalizado, con el monopolio del poder sobre el Estado, hacía inútil el ánimo de formación de fuerzas opositoras, tal como señalan Mainwaring y Scully “Bajo la hegemonía del PRI, la ineficacia del voto desalentaba la participación en las elecciones y la formación de partidos. Sin embargo, la necesidad de realizar elecciones periódicas hacía necesario tener partidos, si bien la combinación de instituciones informales y el recurso al fraude electoral hacían imposible la democracia multipartidista". ${ }^{3}$

La lucha electoral se subordinaba al PRI, quien logro contener a sus opositores en las elecciones presidenciales de 1929, 1940 y 1952 con el uso de la fuerza. En la década de los 70, concretamente con la Reforma Electoral de 1977 cobra importancia la vida electoral y la competencia interpartidista. ${ }^{4}$

En el año 2000, con las elecciones del 02 de julio, se genera un momento crucial en el proceso de democratización mexicano. Según Moreno, dichas elecciones serán recordadas como las Elecciones del cambio en donde, más que una búsqueda clara de políticas públicas o de contenidos programáticos se buscaba el cambio en la alternancia del poder en la presidencia del país. ${ }^{5}$

A partir del año 2000, se generan nuevas alternativas reales de opción electoral, se rompe la única dimensión de gobierno - oposición, en donde tradicionalmente el PRI se alineaba en la dimensión de gobierno y partidos tan disimiles ideológicamente como El Partido de Acción Nacional (PAN) o el Partido de la Revolución Democrática (PRD) podían estar alineados en la dimensión opositora.

La competencia partidista y el rendimiento electoral de los partidos políticos se determina bajo el análisis de una serie de indicadores que demuestran las características del sistema de partidos y el comportamiento electoral de los votantes.

La finalidad de este artículo es revisar mediante una serie de indicadores los posibles alineamientos y realineamientos electorales, en donde se establezca la influencia de otros partidos además del PRI en la arena electoral, indicadores como el Número efectivo de partidos, concen-

\footnotetext{
${ }^{3}$ Mainwaring, Scott y Scully, Timothy, La democracia cristiana en América Latina. Fondo de Cultura Económica. México. 2010 p. 271.

${ }^{4}$ Autores como Sirvent (2001), Moreno (2003) o Dabene (2000),coinciden en señalar que la Reforma Electoral de 1977 marca el principio de la competencia interpartidista en México, pues se genera una apertura hacia otras fuerzas políticas, antes muy débiles, como el Partido de Acción Nacional (PAN). ${ }^{5}$ Moreno, Alejandro. El votante mexicano - Democracia, actitudes políticas y conducta electoral. Fondo de
cultura Económica México. 2003 p. 11.
} 
tración y volatilidad, ayudaran a comprobar y verificar cambios de prefencias electorales de los votantes en escenarios presidenciales con una competencia de partidos real y competitiva.

El primer indicador a tratar en este artículo será el de fragmentación y número efectivo de partidos.

\section{FRAGMENTACIÓN Y NÚMERO EFECTIVO DE PARTIDOS}

La fragmentación es una dimensión importante tal y como señalan Oñate y Ocaña "informa acerca de la medida en la que el poder político se halla disperso o concentrado, esto es, nos permite saber el número de partidos que compiten en el seno de un sistema de partidos" 6 Por tanto, se comprenderá la distribución entre los diferentes partidos y el tipo de competencia que se presenta.

Dicho índice tiene en cuenta tanto el número como el tamaño de los partidos. Según Rae "Representa la probabilidad de que, eligiendo a dos votantes al azar, estos se decanten por partidos diferentes. Al ser una probabilidad, el índice oscila entre 0 y $1^{\prime \prime} 7$ En donde el valor 0 correspondería a un nivel total de abstencionismo y el 1 a una situación en donde un partido político obtuviese el 100 por ciento de los votos, por lo que el índice oscila entre 0,1 y 0,9 . Los valores entre 0,1 y 0,3 corresponden a un régimen unipartidista, entre 0,4 y 0,6 corresponde a un sistema bipartidista, por ejemplo, el 0,5 indica un bipartidismo perfecto, y, entre 0,7 y 0,9 indica la existencia de un sistema multipartidista.

El índice de Fragmentación de Rae, según Sartori, debe ir acompañado por el índice de Numero Efectivo de Partidos, pues se entiende que "la fragmentación debía venir determinada, aunque solo fuera de modo aproximado, por el número de partidos relevantes que existían en su seno, esto es, por el número de partidos con posibilidades de coalición o posibilidades de chantaje. ${ }^{8}$

En relación al debate sobre qué forma es más favorable, entre sistemas bipartidistas o multipartidistas, la literatura tradicional se decanta por el bipartidismo, Lijphart señala que el bipartidismo tiene ciertas ventajas y

\footnotetext{
${ }^{6}$ Oñate, Pablo y Ocaña, Francisco Análisis de datos electorales. Centro de Investigaciones Sociológicas, Cuadernos Metodológicos. Madrid. 1999. p. 36.

${ }^{7}$ RAE, Douglas, The political consequences of electoral laws, New Haven, Yale University Press, 1971.

${ }^{8}$ Sartori, Giovanni, Partidos y sistemas de partido, Madrid Alianza, 1987, p. 157.
} 
criticas. Entre las ventajas del mismo figura "El influjo moderador y central sobre el régimen democrático, los votantes concentrarían su atención en el centro del espectro político, segundo, "el ejecutivo será más estable, puesto no habrá coaliciones de partido" y tercero, "el electorado puede elegir claramente entre dos maneras alternativas de llevar los asuntos públicos" ${ }^{\prime \prime}$ entre las críticas al bipartidismo figura esencialmente en que sus virtudes no son necesariamente atribuibles a su forma, es decir, no por ser bipartidista un régimen, será más inestable su ejecutivo ni el votante concentraría más su atención ni tendría más claridad de atención. ${ }^{10}$

Mainwaring señala que "los sistemas presidencialistas unidos a sistemas multipartidistas presentan notorios problemas de estabilidad institucional".${ }^{11}$ Aunque, ciertamente, la forma presidencial unida a la forma multipartidista cada vez toma más auge en el continente latinoamericano; Chasquetti señala que si un sistema presidencialista viene unido a un sistema multipartidista en donde los partidos puedan hacer coaliciones, entonces, se estabilizaría el sistema democrático. La forma multipartidista, por ende, genera mayor pluralidad y representación sobre los sistemas bipartidistas; ${ }^{12} \mathrm{y}$, en cualquier caso, muchos países del continente están adoptando esta forma como una consecuencia del sistema político actual.

Las variaciones más importantes y significativas de acuerdo a los indicadores que muestran el sistema de partidos y los posibles cambios en el comportamiento electoral, vienen definidas al estudiar las elecciones por Estados. Aspecto que se presenta a continuación.

\footnotetext{
${ }^{9}$ Lijphart, Arend, Las democracias contemporáneas, Ariel Ciencia Política, Barcelona-España, 1998, pp. 123-125.

${ }^{10}$ Para profundizar acerca de los aspectos ventajas y criticas del bipartidismo véase a Blondel (1968), Lijphart (1984), Hurwitz (1971), Siegfried (1956).

${ }^{11}$ Mainwaring, Scott, Presidentialism in Latin America, Latin America Research Review, 1990, p. 25.

12 Chasquetti, Daniel Democracia, multipartidismo y coaliciones en América Latina: evaluando la difícil combinación. Taller de gráficas y servicios. Buenos Aires, Argentina, 2001, p. 2.
} 
Tabla\#1: Índice de fragmentación de RAE y Número efectivo de Partidos en los procesos electorales presidenciales entre 1994 y 2006.

\begin{tabular}{|c|c|c|c|c|c|c|c|c|}
\hline Estad os & $\mathrm{F}=94$ & NEP94 & $\mathrm{F}=\infty$ & NEP00 & $\mathrm{F}=06$ & NEP06 & $\mathrm{F}=\mathrm{x}$ & Nep $=x$ \\
\hline Aguascalientes & 0.65 & 2.8 & 0.59 & 2.4 & 0.67 & 30 & 0.64 & 2.73 \\
\hline Baja California & 0.63 & 2.7 & 0.61 & 2.5 & 0.68 & 3.1 & 0.64 & 2.77 \\
\hline $\begin{array}{l}\text { Baja California } \\
\text { S. }\end{array}$ & 0.60 & 2.5 & 0.69 & 32 & 0.67 & 30 & 0.65 & 2.90 \\
\hline Campeche & 0.64 & 2.7 & 0.65 & 28 & 0.72 & 35 & 0.67 & 3.00 \\
\hline Coahuila & 0.59 & 2.4 & 0.62 & 2.6 & 0.69 & 32 & 0.63 & 2.73 \\
\hline Colima & 0.64 & 2.7 & 0.62 & 2.6 & 0.67 & 30 & 0.64 & 2.77 \\
\hline Chiapas & 0.69 & 3.2 & 0.69 & 32 & 0.67 & 30 & 0.68 & 3.13 \\
\hline Chihuahua & 0.58 & 2.3 & 0.59 & 2.4 & 0.69 & 3.2 & 0.62 & 2.63 \\
\hline Distrito Fed. & 0.71 & 3.4 & 0.68 & 3.1 & 0.59 & 2.4 & 0.66 & 2.97 \\
\hline Durango & 0.66 & 2.9 & 0.62 & 2.6 & 0.68 & 3.1 & 0.65 & 2.87 \\
\hline Guanajuato & 0.63 & 2.7 & 0.55 & 2.2 & 0.59 & 2.4 & 0.59 & 2.43 \\
\hline Guerrero & 0.64 & 2.7 & 0.66 & 29 & 0.64 & 2.7 & 0.65 & 2.77 \\
\hline Hidalgo & 0.61 & 2.5 & 0.66 & 29 & 0.70 & 33 & 0.66 & 2.90 \\
\hline Jalisco & 0.63 & 2.7 & 0.59 & 2.4 & 0.66 & 29 & \begin{tabular}{|l|}
0.63 \\
\end{tabular} & 2.67 \\
\hline Méxi & 0.69 & 3.2 & 0.67 & 3.0 & 0.69 & 32 & 0.68 & 3.13 \\
\hline Michoacán & 0.68 & 3.1 & 0.69 & 32 & 0.67 & 30 & 0.68 & 3.10 \\
\hline Morelos & 0.66 & 2.9 & 0.67 & 30 & 0.68 & 3.1 & 0.67 & 3.00 \\
\hline Nayarit & 0.61 & 2.5 & 0.64 & 2.7 & 0.66 & 29 & 0.64 & 2.70 \\
\hline Nuevo León & 0.61 & 2.5 & 0.59 & 2.4 & 0.65 & 28 & \begin{tabular}{|l|}
0.62 \\
\end{tabular} & 2.57 \\
\hline Oaxaca & 0.66 & 2.9 & 0.68 & 3.1 & 0.66 & 29 & 0.67 & 2.97 \\
\hline Puebla & 0.66 & 2.9 & 0.64 & 2.7 & 0.71 & 3.4 & 0.67 & 3.00 \\
\hline Ouerétaro & 0.59 & 2.4 & 0.61 & 2.5 & 0.66 & 29 & 0.62 & 2.60 \\
\hline Quintana Roo & 0.63 & 2.7 & 0.65 & 28 & 0.71 & 3.4 & 0.66 & 2.97 \\
\hline $\begin{array}{l}\underline{\text { San Luis }} \\
\underline{\text { Potosi }}\end{array}$ & 0.61 & 2.5 & 0.62 & 2.6 & 0.66 & 29 & 0.63 & 2.67 \\
\hline Sinaloa & 0.63 & 2.7 & 0.54 & 2.1 & 0.69 & 32 & \begin{tabular}{|l|}
0.62 \\
\end{tabular} & 2.67 \\
\hline Sonora & 0.66 & 2.9 & 0.60 & 2.5 & 0.64 & 2.7 & 0.63 & 2.70 \\
\hline Tabasco & 0.60 & 2.5 & 0.67 & 30 & 0.55 & 2.2 & 0.61 & 2.57 \\
\hline
\end{tabular}




\begin{tabular}{|l|l|l|l|l|l|l|l|l|}
\hline Tamaulipas & 0.68 & 3.1 & 0.60 & 2.5 & 0.69 & 3.2 & $\mathbf{0 . 6 6}$ & $\mathbf{2 . 9 3}$ \\
\hline T laxcala & 0.64 & 2.7 & 0.66 & 2.9 & 0.67 & 3.0 & $\mathbf{0 . 6 6}$ & $\mathbf{2 . 8 7}$ \\
\hline Veracruz & 0.66 & 2.9 & 0.67 & 3.0 & 0.70 & 3.3 & $\mathbf{0 . 6 8}$ & $\mathbf{3 . 0 7}$ \\
\hline Yucatán & 0.56 & 2.2 & 0.57 & 2.3 & 0.65 & 2.8 & $\mathbf{0 . 5 9}$ & $\mathbf{2 . 4 3}$ \\
\hline Zacatecas & 0.59 & 2.4 & 0.69 & 3.2 & 0.71 & 3.4 & 0.66 & 3.00 \\
\hline Total & $\mathbf{0 . 6 3}$ & $\mathbf{2 7}$ & $\mathbf{0 . 6 3}$ & $\mathbf{2 7}$ & $\mathbf{0 . 6 7}$ & $\mathbf{3 . 0}$ & $\mathbf{0 . 6 4}$ & $\mathbf{2 . 8}$ \\
\hline
\end{tabular}

(Elaboración propia a partir de datos del IFE)

En la anterior tabla se muestran las fragmentaciones de Rae y número efectivo de partidos de Laakso y Taagepera, en donde pueden diferenciarse tres grupos de Estados de acuerdo a sus índices, negrilla se muestran los diez estados que tienen mayor número efectivo de partidos, en gris subrayado los diez Estados que tienen menor índice del mismo y en normal los que están más cercanos a la media total de los tres periodos, en la siguiente tabla se muestra la relación entre número efectivo de partidos y partido ganador en las elecciones de 2006.

Tabla \# 2: Partidos ganadores en Estados con alto y bajo Número efectivo de partidos en las elecciones presidenciales de 2006:13

\begin{tabular}{|c|c|c|c|c|c|}
\hline $\begin{array}{l}\text { Estados con } \\
\text { alto NEP }\end{array}$ & NEP & $\begin{array}{c}\text { Partido } \\
\text { ganador en } \\
2006\end{array}$ & $\begin{array}{c}\text { Estados con } \\
\text { bajo NEP }\end{array}$ & NEP & $\begin{array}{c}\text { Pattido } \\
\text { ganador en } \\
2006\end{array}$ \\
\hline Campeche & 3.00 & PRD & Chihmahum & 2.63 & PAN \\
\hline Chiapas & 3.13 & PRD & Gunnajuato & 2.77 & PAN \\
\hline $\begin{array}{l}\text { Distrito } \\
\text { Fed. }\end{array}$ & 2.97 & PRD & Jalisco & 2.67 & PAN \\
\hline México & 3.13 & PRD & Nuevo León & 2.57 & PAN \\
\hline Michoacín & 3.10 & PRD & Querétam & 2.60 & PAN \\
\hline Monelos & 3.00 & PRD & $\begin{array}{l}\text { SarLLuis } \\
\text { Pot }\end{array}$ & 2.67 & PAN \\
\hline Oaxaca & 2.97 & PRD & Sinaloa & 2.67 & PAN \\
\hline Puebla & 3.00 & PAN & Sordora & 2.70 & PAN \\
\hline Vencruz & 3.07 & PRD & Tabasco & 2.57 & PAN \\
\hline Zacatecas & 3.00 & PRD & Yucatain & 2.43 & PAN \\
\hline
\end{tabular}

(Elaboración propia a partir de datos del IFE y cálculos propios)

\footnotetext{
${ }^{13}$ Se relaciona el número efectivo de partidos y se omite el índice de fragmentación de RAE, pues estos dos índices están fuertemente correlacionados y seria redundante incluir a ambos en las relaciones con los partidos ganadores en 2006.
} 
Se describe una particularidad con respecto al número efectivo de partidos y el partido ganador en las elecciones presidenciales de 2006. El grupo de Estados en donde el número efectivo de partidos supera a la media, predomina notoriamente el PRD y en donde el número efectivo de partidos es inferior a la media existe un predominio del PAN.

Dentro de un sistema electoral competitivo, según Sartori, de tres a cinco partidos como número efectivo de partidos se hablaría de un sistema de "pluralismo moderado" que se caracteriza por "carecer de partidos anti sistemas importantes, carece de oposiciones bilaterales, la distancia ideológica es relativamente pequeña y existe una configuración de coalición bipolar", ${ }^{14}$ este tipo de sistema de partidos es el que se encuentra en los estados en donde predomina el PRD, en donde la mayoría de los votantes se ubicarían en el centro del espectro ideológico. Por otro lado, el mismo autor señala que un sistema de partidos de dos partidos sin llegar el número efectivo de partidos a, al menos tres partidos se hablaría de un sistema de partidos "bipartidista" en donde "dos partidos están en condiciones de competir por la mayoría de los escaños, se reduce la alternativa de coalición, la alternancia o rotación en el poder se da entre dos partidos". ${ }^{15}$ Dentro de este grupo se encuentran los Estados en donde ha ganado el PAN. Para saber si la relación entre número efectivo de partidos y partido ganador es efectiva, se presenta a continuación una correlación momento de Pearson.

Tabla \# 3: Correlación momento de Pearson entre número efectivo de partidos y partido ganador.

\begin{tabular}{|l|c|c|c|c|}
\hline $\begin{array}{l}\text { Corre lación de } \\
\text { Pearson }\end{array}$ & Mediasnep & Winpres 94 & Winpres 00 & Winpres 06 \\
\hline $\begin{array}{l}\text { Mediasnep } \\
\text { (media de } \\
\text { Nume ro efectivo } \\
\text { de partidos) }\end{array}$ & $\mathrm{X}$ & No discrimina & -.045 & $\mathbf{6 2 1}^{* *}$ \\
\hline $\begin{array}{l}\text { Winpres 94 } \\
\text { (ganador 1994) }\end{array}$ & No discrimina & $\mathrm{X}$ & No discrimina & No discrimina \\
\hline $\begin{array}{l}\text { Winpres 00 } \\
\text { (ganador 2000) }\end{array}$ & -.045 & No discrimina & $\mathrm{X}$ & $\mathbf{- . 4 3 4}$ \\
\hline $\begin{array}{l}\text { Winpres 06 } \\
\text { (Ganador 2006) }\end{array}$ & $\mathbf{. 6 2 1}^{\text {** }}$ & No discrimina & $\mathbf{- . 4 3 4}$ & $\mathrm{X}$ \\
\hline
\end{tabular}

(Elaboración propia a partir de datos del IFE)

En la anterior tabla se correlacionan las variables Winpres, que se refieren al partido ganador por Estado en los años 1994, 2000 y 2006, y por otro

${ }^{14}$ Sartori, Giovanni, Parties and Party Systems. A Framework for Analysis. Cambridge University Press, 1976, p. 65.

${ }^{15}$ Idem, 63. 
lado la variable mediasnep referida a la media aritmética del número efectivo de partidos en cada una de las elecciones. Es importante señalar que las elecciones presidenciales del año 1994 no discriminan puesto el PRI ganó en todos los Estados y por tanto no ofrecen correlación.

La correlación entre las variables Winpres06 y mediasnep si es significativa con un valor de 0.621 , es decir, para las elecciones presidenciales del 2006, un alto número efectivo de partidos que es interpretado como un sistema de pluralismo moderado favorece al PRD y a la inversa un sistema de partidos con bajo número efectivo de partidos favorece al PAN.

Este fenómeno encuentra su explicación en que el PAN ha logrado encapsular a sus votantes de acuerdo a criterios de estratificación estatal, al ser un partido con larga trayectoria política ha logrado cooptar votantes dentro de su dimensión ideológica y política, Loaeza describe que el PAN era una organización ideológica construida en torno a una doctrina democristiana y, un partido que atraía votos de protesta de todo tipo, ${ }^{16}$ esto explica su supervivencia, adaptación en la sociedad y encapsulamiento de votantes.

De igual manera el encapsulamiento de algunos Estados por parte del PAN responde a "un efecto neopanista de convertir al partido en un instrumento de representación de los intereses y demandas regionales" ${ }^{17}$

De igual forma se identifica una correlación negativa y medianamente significativa entre winpres00 y winpres06, pero se seguirán buscando posibles explicaciones de esto con otros indicadores que muestren una relación más clara y significativa.

En el gráfico número uno se muestra geográficamente, por Estados, los diferentes niveles, alto - medio - bajo, de Número efectivo de partidos, puede notarse una tendencia en donde, los Estados con un número efectivo de partidos alto se ubica mayoritariamente al sur del país, dichos Estados votaron mayoritariamente al PRD. Los Estados que obtuvieron un NEP medio, se encuentran más dispersos, ubicándose en el centro y norte del país, cabe destacar que en este caso no hay correlación en la supremacía de algún color político y, los estados que tienen un NEP bajo se ubican al igual que el NEP medio en el centro-norte del país.

\footnotetext{
${ }^{16}$ Loaeza, Soledad, El Partido Acción Nacional (PAN) de los márgenes del sistema político al centro del cambio en Mainwaring y Scully Fondo de Cultura Económica, México, 2010, p. 270.

${ }^{17}$ Idem, p. 309.
} 
La ubicación geográfica, para este análisis de NEP, no presenta una relación o tendencia clara dentro de esta investigación, se considera preciso, por ende, acuñar otros análisis de resultados electorales que puedan complementar y ofrecer resultados que clarifiquen el escenario electoral presidencial de la República mexicana. dos. ${ }^{18}$

Gráfico \# 1: Ubicación geográfica según el Número Efectivo de Parti-

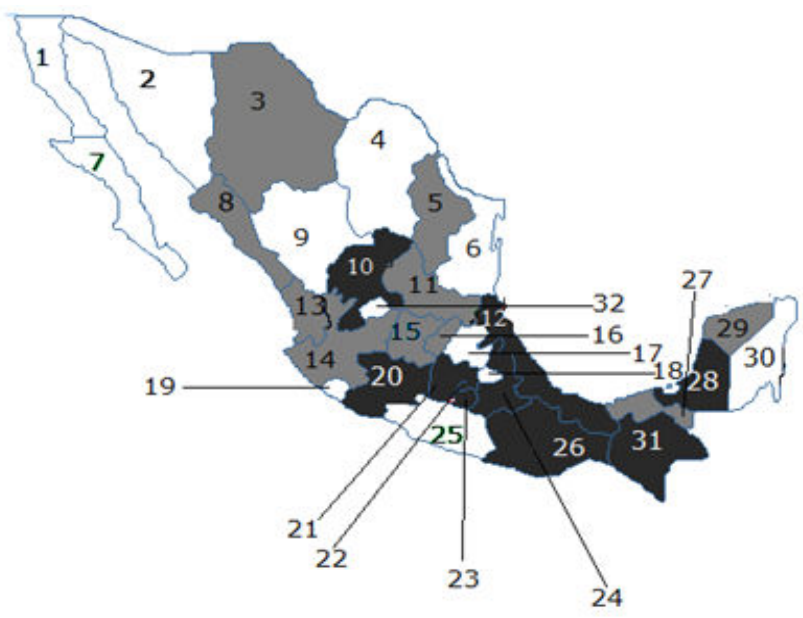

NEP Alto

NEP Medio

NEP Bajo

Es preciso, junto con el número de partidos y sus tamaños respectivos, estudiar el grado en el que el poder político está disperso o concentrado, siendo una dimensión considerablemente relevante. Según Anduiza y Bosch 19 "La concentración del voto es el porcentaje total de votos que suman los dos partidos más votados" ${ }^{20}$ Dicha concentración delimita elementos de competitividad dentro del sistema político estudiado, según Oñate y Ocaña "Las pautas de competición electoral y de gobernabilidad y, así, de estabilidad institucional no serán las mismas en un sistema en el que los dos primeros partidos sumen más del $80 \%$ del voto o de los escaños que en aquel en el que no alcancen el $40 \% " 21$ en el primer caso estaríamos hablando de un sistema electoral fuerte, capaz de orientar el voto y reducir el número de partidos relevantes y en el segundo caso, si la concentración es menor porcentualmente, se hablaría de un sistema electoral débil.

\footnotetext{
18 32-Aguascalientes, 1-Baja California, 7-Baja California S., 28-Campeche, 4-Coahuila, 19-Colima, 31-Chiapas 3-Chihuahua, 22-Distrito Fed. , 9-Durango, 15-Guanajuato, 25-Guerrero, 17-Hidalgo, 14Jalisco, 21-México, 20-Michoacán , 23-Morelos, 13-Nayarit, 5-Nuevo León, 26-Oaxaca , 24-Puebla, 16Querétaro , 30-Quintana Roo , 11-San Luis Potosí , 8-Sinaloa,2-Sonora,27-Tabasco, 6-Tamaulipas, 18Tlaxcala, 12-Veracruz , 29-Yucatán, 10-Zacatecas.

${ }^{19}$ Anduiza, Eva y Bosch, Agustí, Comportamiento político y electoral. Ariel ciencia Política. Barcelona España, 2004. p. 89.

${ }^{20}$ La formula de concentración es Concentración $=(\%$ voto $A)+(\%$ voto $B)$. en donde A y B son los partidos más votados.

${ }^{21}$ Oñate, Pablo y Ocaña, Francisco, op cit., 39.
} 
Luis Eduardo León Ganatios

\begin{tabular}{|c|c|c|c|c|}
\hline Estados & $\begin{array}{c}\text { Concentración } \\
1994\end{array}$ & $\begin{array}{c}\text { Concentración } \\
2000\end{array}$ & $\begin{array}{c}\text { Concentración } \\
2005\end{array}$ & $\begin{array}{c}\text { Media de } \\
\text { oncentración }\end{array}$ \\
\hline Aguascalientes & 83.10 & 87.82 & 70.33 & 80.42 \\
\hline B aja California & 85.10 & 86.80 & 70.94 & 80.95 \\
\hline Baja Califomia S. & 87.42 & 69.66 & 77.41 & 78.16 \\
\hline Campeche & 74.61 & 80.81 & 64.23 & 7322 \\
\hline Chiapas & 77.11 & 69.54 & 76.94 & 74.53 \\
\hline Chihuahua & 86.32 & 89.54 & 74.53 & 83.46 \\
\hline Coalhwila & 84.31 & 87.04 & 69.56 & 80.30 \\
\hline Colima & 80.32 & 85.24 & 71.46 & 79.01 \\
\hline Distrito Fed. & 68.97 & 69.60 & 85.52 & 74.70 \\
\hline Durango & 77.68 & 86.13 & 71.54 & 78.45 \\
\hline Guanajuato & 82.99 & 88.65 & 77.73 & 83.12 \\
\hline Guerrero & 82.25 & 77.91 & 77.94 & 79.37 \\
\hline Hidalgo & 75.81 & 78.10 & 67.42 & 7378 \\
\hline Ialisco & 85.61 & 88.97 & 73.58 & 8272 \\
\hline México & 71.97 & 75.6 & 74.38 & 74.01 \\
\hline Michoacán & 78.48 & 67.32 & 75.66 & 73.82 \\
\hline Morelos & 72.26 & 75.73 & 76.03 & 74.67 \\
\hline Nayarit & 75.72 & 78.74 & 75.52 & 76.66 \\
\hline Nuevo León & 87.81 & 89.82 & 76.50 & 8471 \\
\hline O axaca & 77.13 & 69.19 & 77.68 & 74.67 \\
\hline Puebla & 76.01 & 83.12 & 69.73 & 76.29 \\
\hline O uerétaro & 86.92 & 86.32 & 73.20 & 82.15 \\
\hline Quintana Roo & 81.53 & 79.11 & 67.23 & 75.96 \\
\hline San Luis Potosí & 82.20 & 86.48 & 70.39 & 79.69 \\
\hline Sinaloa & 80.97 & 87.77 & 67.83 & 78.86 \\
\hline Sonora & 79.93 & 84.41 & 75.82 & 80.05 \\
\hline Tabaco & 86.69 & 70.77 & 94.09 & 83.85 \\
\hline Tamaulipas & 72.72 & 88.01 & 67.76 & 76.16 \\
\hline Tlaxcala & 76.65 & 71.94 & 78.16 & 75.58 \\
\hline Veracrux & 74.63 & 77.60 & 69.44 & 73.89 \\
\hline Yucatán & 92.88 & 93.18 & 79.13 & 88.40 \\
\hline Zacatecas & 82.68 & 72.19 & 67.57 & 74.15 \\
\hline T otal & 80.27 & 80.72 & 73.91 & 78.30 \\
\hline
\end{tabular}

(Tabla de elaboración propia en base a la fórmula de concentración calculada por Estados)Los Estados identificados con el color gris subrayado, son los que están por debajo de la media total que es 78.30, los Estados 
identificados con el color gris son cercanos a la media y los identificados con el color negritas están por encima de la media, a continuación se relaciona el tipo de concentración con el partido ganador en las elecciones presidenciales de 2006.

Tabla \# 5: Partidos ganadores en Estados con alto y bajo índice de concentración en las elecciones presidenciales de 2006:

\begin{tabular}{|c|c|c|c|c|c|}
\hline $\begin{array}{l}\text { Estados con alta } \\
\text { Conce ntración }\end{array}$ & Concentración & $\begin{array}{l}\text { Partido } \\
\text { ganador }\end{array}$ & $\begin{array}{c}\text { Estados con } \\
\text { baja }\end{array}$ & Concentración & $\begin{array}{l}\text { Partido } \\
\text { ganador }\end{array}$ \\
\hline Aguascalientes & 80.42 & $\begin{array}{l}\text { en } 2006 \\
\text { PAN }\end{array}$ & $\begin{array}{l}\text { Concentración } \\
\text { Campeche }\end{array}$ & $\pi 3.22$ & $\begin{array}{c}\text { en } 2006 \\
\text { PRD }\end{array}$ \\
\hline Baja California & 80.95 & PAN & Chiapas & 74.53 & PRD \\
\hline Chihuahua & 83.46 & PAN & Distrito Fed. & 74.70 & PRD \\
\hline Coahuila & 80.30 & PAN & Hidalgo & 73.78 & PRD \\
\hline Guanajuato & 83.12 & PAN & México & 74.01 & PRD \\
\hline Ialisco & 82.72 & PAN & Micho acán & 73.62 & PRD \\
\hline Nuevo León & 84.71 & PAN & Monelos & 74.67 & PRD \\
\hline Q werétano & 82.15 & PAN & Oaxaca & 74.67 & PRD \\
\hline$\underline{\text { Thasco }}$ & 83.85 & PAN & Vexacrux & 73.89 & PRD \\
\hline Yucatán & 88.40 & PAN & Zacatecas & 74.15 & PRD \\
\hline
\end{tabular}

(Fuente: Elaboración propia a partir de datos del IFE)

Se observa una relación directa entre el índice de concentración y partido ganador, relación por demás lógica, siendo preciso mostrar la correlación entre partido ganador e índice de concentración, la cual debe ser significativa por la relación descriptiva observada.

De igual manera se puede presumir una correlación significativa y negativa entre concentración y Número efectivo de partidos, entendida bajo la siguiente hipótesis; a mayor concentración del voto menor es el número efectivo de partidos. $Y$, en segundo término, en donde ocurre esta situación, para el caso de México, habrá predominio de un partido político concreto. 
Tabla \# 6: Correlación momento de Pearson entre índice de concentración y partido ganador.

\begin{tabular}{|l|c|l|c|c|}
\hline $\begin{array}{l}\text { Correlación de } \\
\text { Pearson }\end{array}$ & Mediasnep & Winpres 94 & Winpres 00 & Winpres 06 \\
\hline $\begin{array}{l}\text { Mediac oncen } \\
\text { (media de } \\
\text { Concentración) }\end{array}$ & $\mathrm{X}$ & No discrimina & 211 & $-.77^{\text {*t }}$ \\
\hline $\begin{array}{l}\text { Winpres 94 } \\
\text { (ganador 1994) }\end{array}$ & No discrimina & $\mathrm{X}$ & No discrimina & No discrimina \\
\hline $\begin{array}{l}\text { Winpres 00 } \\
\text { (ganador 2000) }\end{array}$ & .211 & No discrimina & $\mathrm{X}$ & -.275 \\
\hline $\begin{array}{l}\text { Winpres 06 } \\
\text { (Ganador 2006) }\end{array}$ & $-.767^{*+}$ & No discrimina & -.275 & $\times$ \\
\hline
\end{tabular}

(Elaboración propia a partir de la media de concentración y ganador en 2006).

Tabla \# 7: Correlación momento de Pearson entre índice de concentración y número efectivo de partidos.

\begin{tabular}{|l|c|c|}
\hline \multicolumn{1}{|c|}{ Medias } & mediasnep & mediconcen \\
\hline mediasnep & $\mathrm{X}$ & $-.914^{*+}$ \\
\hline medic oncen & $-.914^{*+}$ & $X$ \\
\hline
\end{tabular}
NEP).

(Elaboración propia a partir de la media de concentración y media de

La correlación negativa descrita en la anterior correlación momento de Pearson, demuestra la hipótesis enunciada y para el caso concreto de México muestra que el PAN se ve favorecido en escenarios de alta concentración y bajo número efectivo de partidos, esto demuestra que el PAN concentra preferencias cautivas, $y$, que en los Estados donde ejerce dominio electoral sólo un partido suele alinearse en su contra.

Por otra parte el PRD se favorece de escenarios menos polarizados, con baja concentración y número efectivo de partidos alto, lo cual su dominio electoral suscita una competitividad más enunciada con más partidos políticos. El PRD no tiene votos cautivos, las preferencias de los votantes, circunstancialmente pueden cambiar al otro partido que comparte dimensión ideológica con este, el PRI. 
Gráfico \# 2: Ubicación geográfica según el Índice de Concentración de Partidos. ${ }^{22}$
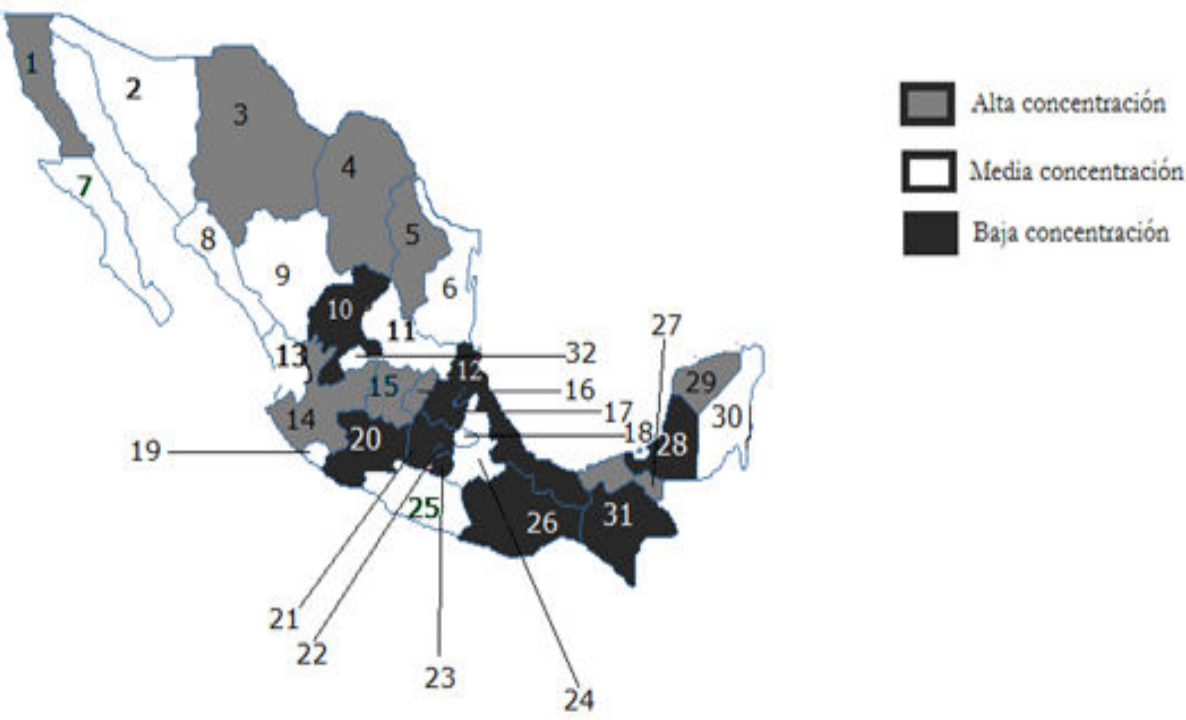

\section{VOLATILIDAD ELECTORAL}

La volatilidad electoral es un indicador que muestra el cambio de una elección con respecto a la anterior, "Este índice ${ }^{23}$ indica los cambios mínimos que se han debido producir entre dos elecciones para que se verifique el cambio de la segunda con respecto a la primera. En el caso de una estabilidad perfecta entre dos elecciones la volatilidad total será de 0 (cero) o muy cercano a este" ${ }^{24}$

Representa una posibilidad dinámica de estudio de los partidos, sistemas de partidos, inclusive entre bloques de partidos definidos, por ejemplo izquierda y derecha, y por tanto, comprobar la probable existencia de cambios interelectorales.

22 32-Aguascalientes, 1-Baja California, 7-Baja California S., 28-Campeche, 4-Coahuila, 19-Colima, 31Chiapas 3-Chihuahua, 22-Distrito Fed., 9-Durango, 15-Guanajuato, 25-Guerrero, 17-Hidalgo, 14Jalisco, 21-México, 20-Michoacán , 23-Morelos, 13-Nayarit, 5-Nuevo León, 26-Oaxaca, 24-Puebla, 16Querétaro, 30-Quintana Roo, 11-San Luis Potosí, 8-Sinaloa,2-Sonora, 27-Tabasco, 6-Tamaulipas, 18Tlaxcala, 12-Veracruz, 29-Yucatán, 10-Zacatecas.

${ }^{23}$ La volatilidad se calcula mediante la siguiente fórmula (Volatilidad) $\mathrm{V}=(\mathrm{Vi2}-\mathrm{Vi1}) / 2$ Siendo $V i 1$ el porcentaje de votos de un partido en la elección primera y $V i 2$ el porcentaje de votos del mismo partido en la siguiente elección. Pedersen, Mogens Changing patterns of electoral volatility in European party systems. London. Sage. 1983.

${ }^{24}$ Anduiza, Eva y Bosch, Agustí, op cit., p. 93. 
Según Oñate y Ocaña "Esta dimensión nos permite conocer el grado en el que el sistema de partidos está cristalizado o no, si es estable y si la o las líneas en torno a las cuales ha venido articulándose siguen siendo o no relevantes, permite conocer la medida y el calado del cambio electoral" $(1999,44)$.

$\mathrm{Al}$ analizar los resultados de dos elecciones consecutivas, se pueden presentar diversos cambios, una tipología apropiada, a juicio de esta investigación, es la propuesta por Harrop y Miller en donde pueden producirse dos alternativas; un cambio en el resultado (elecciones críticas o desviadas) $\mathrm{y}$, continuidad en el resultado (elecciones de conversión o elecciones de continuidad)..$^{25}$

En el primer caso, las elecciones críticas o de realineamiento se producen cuando cambian las bases de apoyo de los partidos como de sus resultados, en las elecciones desviadas cambian los resultados de los partidos pero no sus bases de apoyo, esto puede medirse cuando la volatilidad es alta y, cuando las volatilidades arrojan signo negativo. En el segundo caso, las elecciones de continuidad, se producen cuando existen pocos cambios, en las elecciones de conversión cambian las bases de apoyo pero el resultado electoral del partido se mantiene constante, esto puede medirse con una baja volatilidad cercana a cero y signo positivo en los resultados, o signo negativo pero muy cercano a cero.

Tabla \# 8: Índice de volatilidad en las elecciones presidenciales entre los años 2000 y 2006:

\begin{tabular}{|l|c|c|c|c|}
\hline Estados & $\begin{array}{c}\text { Volatilidad PRI } \\
-00 / 06\end{array}$ & $\begin{array}{c}\text { Volatilidad } \\
\text { PAN - 00/06 }\end{array}$ & $\begin{array}{c}\text { Volatilidad } \\
\text { PRD -00/06 }\end{array}$ & Total \\
\hline Aguascalientes & -5.17 & -3.58 & 7.37 & $\mathbf{1 6 . 1 2}$ \\
\hline Baja Califomia & -7.83 & -1.20 & 7.31 & $\mathbf{1 6 . 3 4}$ \\
\hline Baja Califomia & -8.47 & -0.93 & 8.08 & $\mathbf{1 7 . 4 8}$ \\
\hline Sur & & & & \\
\hline Campeche & -6.40 & -4.10 & 9.47 & $\mathbf{1 9 . 9 7}$ \\
\hline Chiapas & -4.76 & -4.76 & 9.19 & $\mathbf{1 8 . 7 1}$ \\
\hline Chihulahua & -5.72 & -1.79 & 5.73 & $\mathbf{1 3 . 2 4}$ \\
\hline Coahuila & -5.86 & -2.88 & 7.37 & $\mathbf{1 6 . 1 1}$ \\
\hline Colima & -3.59 & -3.30 & 6.60 & $\mathbf{1 3 . 4 9}$ \\
\hline Distrito Fed. & -7.73 & -8.13 & $\mathbf{1 6 . 0 9}$ & $\mathbf{3 1 . 9 5}$ \\
\hline Durango & -8.65 & 1.35 & 6.25 & $\mathbf{1 6 . 2 5}$ \\
\hline
\end{tabular}

${ }^{25}$ Harrop, M. y Miller, WL, Elections and voters, Londres Mcmillan 1987, p. 79. 


\begin{tabular}{|l|c|c|c|c|}
\hline Guanajuato & -4.54 & -0.93 & 4.41 & 9.88 \\
\hline Guerrero & -8.08 & -1.21 & 8.09 & 17.38 \\
\hline Hidalgo & -9.28 & -3.99 & 12.03 & 25.3 \\
\hline Jalisco & -5.82 & -1.88 & 6.50 & 14.2 \\
\hline México & -6.92 & -6.32 & 12.27 & 25.51 \\
\hline Michoacán & -5.62 & 2.93 & 2.02 & 10.57 \\
\hline Morelos & -7.32 & -6.75 & 12.33 & 26.4 \\
\hline Nayanit & -7.47 & -5.61 & 12.06 & 25.14 \\
\hline Nuevo León & -6.30 & -0.36 & 4.83 & 11.49 \\
\hline Oaxaca & -5.50 & -4.85 & 10.57 & 20.92 \\
\hline Puebla & -8.70 & -2.52 & 10.06 & 21.28 \\
\hline Querétaro & -7.11 & -1.52 & 8.61 & 17.24 \\
\hline Quintana Roo & -2.83 & -8.66 & 10.35 & 21.84 \\
\hline San Lưis Potosí & -8.62 & 0.57 & 6.40 & 15.59 \\
\hline Sinaloa & -18.56 & 6.65 & 10.73 & 35.94 \\
\hline Sonora & -7.42 & -0.34 & 6.35 & 14.11 \\
\hline Tabasco & -0.82 & 15.35 & -13.91 & 30.08 \\
\hline Tamaulipas & -7.32 & -3.08 & 9.08 & 19.48 \\
\hline Thaxcala & -10.94 & -0.67 & $\mathbf{1 0 . 2 4}$ & 21.85 \\
\hline Veracnuz & -6.49 & -2.84 & 8.42 & $\mathbf{1 7 . 7 5}$ \\
\hline Yucatárn & -6.56 & -0.46 & 5.98 & $\mathbf{1 3 . 0}$ \\
\hline Zacatecas & -7.18 & -0.72 & 6.27 & $\mathbf{1 4 . 1 7}$ \\
\hline Total & $\mathbf{6 . 9 8}$ & $\mathbf{3 . 4 4}$ & 8.59 & $\mathbf{1 9 . 0 2}$ \\
\hline & & & & \\
\hline & & & & \\
\hline
\end{tabular}

(Elaboración propia a raíz de datos del IFE y aplicación de la formula de volatilidad de Pedersen).

La suma de los totales de volatilidad por Estados se ha hecho sin tomar en cuenta el signo, de igual forma se ha hecho con los totales por partidos, se han resaltado con color negritas los Estados con mayor índice de volatilidad, en negro, aquellos Estados que tienen una media cercana al total de la media y, en gris subrayado, aquellos que tienen una baja volatilidad, si un partido tiene una alta volatilidad y mayor aún en signo negativo se puede hablar de elecciones criticas, como por ejemplo el PRI en Sinaloa y el PRD en Tabasco, de elecciones desviadas se pueden ver ejemplos con el PAN en Tabasco, el PRD en distrito Federal, Morelos y Nayarit.

Un ejemplo de elecciones de continuidad se pueden apreciar con el PAN en los Estados de Baja California Sur, Guanajuato, Nuevo León, San Luís Potosí, Sonora, Tlaxcala, Yucatán y Zacatecas y, elecciones de conver- 
sión se pueden apreciar en casi todos los resultados obtenidos por el PRI para el 2006.

Tabla \#9: Partidos ganadores en Estados con alto y bajo índice de volatilidad en las elecciones presidenciales de 2006:

\begin{tabular}{|c|c|c|c|c|c|}
\hline $\begin{array}{l}\text { Estados alta } \\
\text { Volatilidad }\end{array}$ & Media & $\begin{array}{l}\text { Pattido } \\
\text { ganador }\end{array}$ & $\begin{array}{c}\text { Estados baja } \\
\text { Volatilidad }\end{array}$ & Media & $\begin{array}{l}\text { Pattido } \\
\text { ganador }\end{array}$ \\
\hline $\begin{array}{l}\text { Distrito } \\
\text { Fed. }\end{array}$ & 31.95 & PRD & Chitruahua & 13.24 & PAN \\
\hline Hidalgo & 25.3 & PRD & Coahurila & 16.11 & PAN \\
\hline México & 2551 & PRD & Colima & 13.49 & PAN \\
\hline Morelos & 26.4 & PRD & Guarnajuato & 9.88 & PAN \\
\hline Nayanit & 25.14 & PRD & Jalisco & 14.2 & PAN \\
\hline Oaxaca & 20.92 & PRD & Michoacán & 10.57 & PRD \\
\hline Puebla & 21.28 & PAN & Nuevo León & 11.49 & PAN \\
\hline $\begin{array}{l}\text { Quintana } \\
\text { Roo }\end{array}$ & 21.84 & PRD & $\begin{array}{l}\text { San Luis } \\
\text { Potosi }\end{array}$ & 15.59 & PAN \\
\hline Simaba & 35.94 & PAN & Sonota & 14.11 & PAN \\
\hline Tabasco & 30.08 & PAN & Yucatán & 13.0 & PAN \\
\hline Thaxcala & 21.85 & PRD & Zacatecas & 14.17 & PRD \\
\hline
\end{tabular}

(Fuente: Elaboración propia a partir de datos del IFE)

Puede observarse en la anterior tabla que los Estados con alta volatilidad electoral son dominados mayoritariamente por el PRD, caso contrario ocurre con los Estados con baja volatilidad en donde predomina el PRD.

Es preciso realizar una correlación momento de Pearson para determinar la relación existente entre volatilidad y partido ganador de las elecciones presidenciales de 2006. 
Tabla \# 10: Correlación momento de Pearson entre índice de volatilidad y partido ganador.

\begin{tabular}{|l|c|c|c|c|}
\hline $\begin{array}{l}\text { Corre laciones } \\
\text { Winpress }\end{array}$ & $\begin{array}{c}\text { Winpress } \\
\mathrm{X}\end{array}$ & $\begin{array}{c}\text { Volapri0006 } \\
\text { No } \\
\text { sigrificativa }\end{array}$ & $\begin{array}{c}\text { Volapan0006 } \\
-.436^{*}\end{array}$ & $\begin{array}{c}\text { Volaprd0006 } \\
.414^{*}\end{array}$ \\
\hline $\begin{array}{l}\text { Volapri0006 (Volatilidad } \\
\text { PRI de los años 2000y } \\
\text { 2006) }\end{array}$ & $\begin{array}{c}\text { No } \\
\text { significativa }\end{array}$ & $\mathrm{X}$ & $\begin{array}{c}\text { No } \\
\text { significativa }\end{array}$ & $-.470^{*}$ \\
\hline $\begin{array}{l}\text { Volapan0006(Volatilidad } \\
\text { PAN de los años 2000y } \\
\text { 2006) }\end{array}$ & $-.436^{*}$ & $\begin{array}{c}\text { No } \\
\text { sigrificativa }\end{array}$ & $\mathrm{X}$ & $-.829^{* *}$ \\
\hline $\begin{array}{l}\text { Volaprd0006(Volatilidad } \\
\text { PRD de los años 2000y } \\
\text { 2006) }\end{array}$ & $.414^{*}$ & $-.470^{*}$ & $-.829^{* *}$ & $\mathrm{X}$ \\
\hline
\end{tabular}

(Elaboración propia).

En la correlación de Pearson se pueden observar ciertos movimientos electorales interesantes, el PRD obtiene correlación negativa tanto con el PAN como con el PRI, es decir, a medida que crece el voto perredista disminuye el voto panista, priista y viceversa. Si se toman en cuenta las auto ubicaciones ideológicas de los partidos políticos el PAN y el PRI están más cercanos ideológicamente, el PAN con un 6,23 y el PRI con un 4,87 frente al PRD con un 2,37. ${ }^{26}$ (Alcántara, 2006,343) entonces la correlación es lógica y esperada. Sin embargo, el PAN y el PRI pertenecen a diferentes dimensiones ideológicas de izquierda y derecha ${ }^{27}$ lo que hace discutible esta inercia de transferencia de voto entre los partidos PAN y PRI.

${ }^{26}$ Alcántara, Manuel Partidos políticos latinoamericanos ¿Instituciones o maquinas ideológicas? Ediciones Gernika México, D.F. 2006 p. 343.

${ }^{27}$ Dentro de una escala de valores que designa la dimensión izquierda - derecha y centro, en la mayoría de los casos se ordenan del 1 al 10 o del cero al 10, en donde desde el cero o uno hasta el 4 ó 5 corresponden a la dimensión de izquierda, entre el 5 o el 6 una posición central y desde el 6 hasta el 10 la dimensión de derecha. León Ganatios, Luis Crisis y cambio de la partidocracia tradicional en las elecciones presidenciales de 1998, una aplicación de la teoría direccional del voto. Tesis doctoral. Universidad de Salamanca. Salamanca-España, 2005, p. 41. 
Gráfico \# 3: Ubicación geográfica según el Índice de Volatilidad de Partidos. $^{28}$

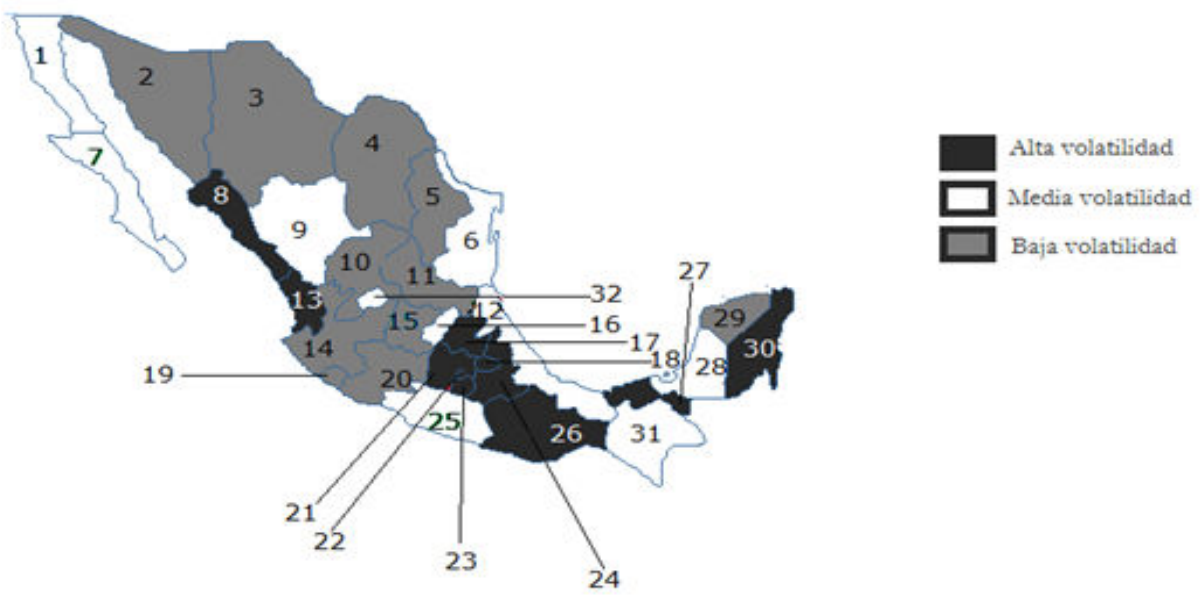

CONCLUSIONES:

El escenario electoral político de México ha cambiado en las últimas tres elecciones, según Magaloni, ${ }^{29}$ en el año de 1994 el PRI "seguía siendo un partido predominante y el PAN o el PRD se alineaban en busca de ser la segunda fuerza electoral" rol que tomo el PAN en ese año. A partir del año 2000 entra un escenario de alternabilidad en el poder y el PAN consigue ganar dos elecciones consecutivas (2000 y 2006) y el PRD logra ubicarse como segunda fuerza para las elecciones de 2006. Incluso dejando al PRI sin obtener la victoria en ninguno de los 32 estados de la República mexicana.

Según el índice de número efectivo de partidos y el de concentración que se correlacionan negativamente, el dominio electoral del PAN sucede en Estados con una alta concentración y un bajo número efectivo de partidos, al igual que una baja volatilidad. Esto se traduce en que el PAN ha conseguido cooptar a los votantes, para crear preferencias más estables y continuas, de los Estados según el análisis de sus datos electorales. El PAN de acuerdo a la geografía electoral mostrada tiene fuerte arraigo en los estados de Chihuahua, Jalisco, Guanajuato, Yucatán y Coahuila.

Caso contrario ocurre con el PRD, que es más efectivo en Estados con

\footnotetext{
28 32-Aguascalientes, 1-Baja California, 7-Baja California S., 28-Campeche, 4-Coahuila, 19-Colima, 31Chiapas, 3-Chihuahua, 22-Distrito Fed., 9-Durango, 15-Guanajuato, 25-Guerrero, 17-Hidalgo, 14Jalisco, 21-México, 20-Michoacán, 23-Morelos, 13-Nayarit, 5-Nuevo León, 26-Oaxaca, 24-Puebla, $16-$ Querétaro, 30-Quintana Roo, 11-San Luis Potosí, 8-Sinaloa,2-Sonora, 27-Tabasco, 6-Tamaulipas, 18Tlaxcala, 12-Veracruz, 29-Yucatán, 10-Zacatecas.

${ }^{29}$ Magaloni, Beatriz, Dominio de partido y dilemas duvergerianos en las elecciones presidenciales de 1994 en
México. Política y gobierno. Volumen III, número 2, 1996, México.
} 
una baja concentración, un elevado índice de número efectivo de partidos y una alta volatilidad, lo que significa que el PRD ha aprovechado escenarios más críticos en el orden electoral. Y, domina para las elecciones de 2006 los estados de Oaxaca, Campeche, México, Morelos y Puebla.

No teniendo un claro dominio de color político el resto de Estados.

Sin embargo, el PRI toma un papel predominante, a pesar de no haber obtenido la victoria electoral en ninguno de los 32 estados en las elecciones presidenciales de 2006. La volatilidad del PRI, correlacionada negativamente con la del PAN, demuestra que el PRI puede cooptar al votante panista, una transferencia de voto que puede darse por descontento o desarraigo del votante panista, dicho votante desarraigado del PAN no lo podría cooptar el PRD. De igual forma, el PRI, por su situación de centro ideológico, puede cooptar a los votantes del PRD, cosa que tampoco puede hacer el PAN.

Toda esta situación deja un escenario algo favorable para el PRI, a pesar de no haber ganado en ningún Estado en las elecciones de 2006, para las elecciones presidenciales de 2012, el PAN buscaría su fuerza en la concentración de sus votantes y, el PRD necesitaría fuertes alianzas de unión para no perder un electorado, que nunca ha estado realmente cooptado electoralmente para sus intereses.

\section{BIBLIOGRAFÍA}

Baez, Carlos, México en Alcántara Manuel y Freidenberg Flavia (Coord.) Partidos Políticos de América Latina. Fondo de cultura económica (México) 2003. p. 63.

Moreno, Alejandro, El votante mexicano - Democracia, actitudes políticas y conducta electoral, Fondo de cultura Económica México, 2003.

Oñate, Pablo y Ocaña Francisco, Análisis de datos electorales, Centro de Investigaciones Sociológicas, Cuadernos Metodológicos, Madrid. 1999.

Rae, Douglas, The political consequences of electoral laws, New Haven, Yale University Press, 1971.

Sartori, Giovanni, Partidos y sistemas de partido, Madrid Alianza, 1987. 
Lijphart, Arend, Las democracias contemporáneas, Ariel Ciencia Política, Barcelona, España, 1998.

Mainwaring, Scott, Presidentialism in Latin America, Latin America Research Review, 25. 1990.

Chasquetti, Daniel, Democracia, multipartidismo y coaliciones en América Latina: evaluando la difícil combinación, Taller de gráficas y servicios, Buenos Aires, Argentina, 2001.

Sartori, Giovanni, Parties and Party Systems. A Framework for Analysis, Cambridge, University Press, 1976.

Loaeza, Soledad, El partido acción nacional (PAN) de los márgenes del sistema político al centro del cambio en Mainwaring, Scott y Scully, Timothy, La democracia cristiana en América Latina, Fondo de Cultura Económica. México, 2010.

Anduiza, Eva y Bosch, Agustí, Comportamiento político y electoral, Ariel ciencia Política, Barcelona, España, 2004.

Pedersen, Mogens, Changing patterns of electoral volatility in European party systems, London, Sage, 1983.

Harrop, M. y Miller, WL, Elections and voters, Londres Mcmillan, 1987.

Alcantara, Manuel, Partidos políticos latinoamericanos ¿Instituciones o maquinas ideológicas? Ediciones Gernika, México D.F., 2006.

León Ganatios, Luis, Crisis y cambio de la partidocracia tradicional en las elecciones presidenciales de 1998, una aplicación de la teoría direccional del voto, Tesis doctoral, Universidad de Salamanca, Salamanca, España, 2005.

Magaloni Kerpel, Beatriz, Dominio de partido y dilemas duvergerianos en las elecciones presidenciales de 1994 en México. Política y gobierno, volumen III, número 2, 1996, México.

Dabene, Olivier, América Latina en el siglo XX, Editorial Síntesis, Madrid, 1999.

González Sánchez, José, Para entender nuestra democracia. La consolidación del sistema de partidos en México, Ediciones GERNIKA, México, D.F., 2006. 
INSTITUTO FEDERAL ELECTORAL (2011) Atlas electoral. [Disponible en] http://www.ife.org.mx/documentos/RESELEC/SICEEF/principal.html

Mayer-Serra, Carlos y Nacif, Benito, Lecturas sobre el cambio político en México, Centro de Investigación y docencia Económicas (CIDE), 2002.

Sirvent, Carlos, Alternancia y distribución del voto en México, Ediciones GERNIKA, México, D.F., 2001. 
